\title{
Análisis sociohistórico de las relaciones entre el conflicto social y las innovaciones tecnológicas
}

\author{
Belén CASAS Mas \\ Universidad Complutense de Madrid \\ bcasasmas@gmail.com
}

\begin{abstract}
Resumen:
Vivimos en una sociedad globalizada y sustentada por las TIC, en las que las dinámicas entre la comunicación pública y el orden social (consenso y conflicto social), han cambiado, están cambiando, y continuarán haciéndolo en el futuro. El propósito de este estudio es contribuir al conocimiento de estos cambios. Pero en la medida en la que sean otros los efectos que la comunicación pública genera, tendrá que ser distinto el uso que se haga de la comunicación en las políticas públicas. Con este trabajo se pretende analizar las transformaciones en la comunicación pública en la era de la globalización que influyeren en el orden social. El estudio explora una de las dimensiones que se incluyen en la Investigación: "La Producción Social de la Comunicación y la Reproducción Social en la Era de la Globalización”, realizada por el Grupo de investigación de la UCM Identidades sociales y comunicación. De esta forma, el trabajo que se presenta en este artículo parte de un diseño previo macrosociológico, sociohistórico y dirigido a la transferencia de resultados.
\end{abstract}

Palabras clave: Globalización; orden social; consenso; conflicto; comunicación pública.

\section{A socio-historical analysis of the relations between social conflict and technological innovations}

\begin{abstract}
:
We are living in a globalised society, underpinned by ICT, where the dynamics between public communication and social order (social consensus/conflict] have changed, are changing and will do so again in the future. The purpose of this study is therefore to contribute to the understanding of these changes. But to the extent that the public communication generates other effects, the use of communication in the public policies will have to be different. The aim is to analyse public communication transformations in the globalisation era that influence social order. The study explores one of the dimensions of the Research: "Social Production Communication and Social Reproduction in the Globalisation Era", carried out by the UCM Group research: Social identities and communication. Consequently, the work presented in this article is based on its previous design, which is macrosociological, sociohistorical and directed at the transference of the results.
\end{abstract}

Key Words: Globalisation; social order; consensus; conflict; public communication.

Referencia normalizada:

Casas-Mas, B. (2014): Análisis sociohistórico de las relaciones entre el conflicto social y las innovaciones tecnológicas. Historia y Comunicación Social. Vol. 19. Núm. Especial Febrero. Págs. 249-260.

Sumario: 1. Introducción. 2. Estado de la cuestión y justificación del objeto de estudio. 3. Metodología. 4. Mecanismos de reproducción social en la era de la globalización: principios teóricos. 5. Información relevante extraída sobre el consenso y conflicto social. 6 . A modo de conclusión 


\section{Introducción}

Los cambios comunicativos, especialmente, la aparición de las TIC, están jugando un papel fundamental en la alteración o el mantenimiento del orden social. Es por eso que se considera pertinente profundizar en el papel de la comunicación en el cambio y la reproducción social. Este ha sido uno de los grandes tópicos al que sociólogos y estudiosos han prestado mucha atención a lo largo de la historia; sin embargo, parece necesario y, quizás hoy más que nunca, indagar en los vínculos que existen entre la comunicación y sus tecnologías, así como en la gestión del consenso y el conflicto social, en la era de la globalización, puesto que estos vínculos conforman un conocimiento básico para el diseño de políticas sociales de vital importancia.

De esta forma, se considera de rigor el recurso a la comunicación pública cuando se quiere prevenir o tratar los conflictos sociales como, por ejemplo, el desarrollo de programas para la prevención de anomias (trastornos de la alimentación, drogadicción...), campañas contra la violencia de género, programas de integración social, etc. Del mismo modo, cuando hay que reforzar el consenso resulta imprescindible el recurso a la comunicación pública, por ejemplo, cuando se trata de fortalecer la cohesión endogrupal frente al terrorismo o la segregación, o cuando hay que promover actividades solidarias en situaciones de catástrofes y desastres naturales, etc.

Estos son solo algunos ejemplos que ilustran la importancia de la comunicación pública en el mantenimiento del orden social, precisamente, el ámbito de estudio en el que se enmarca el presente artículo. Se trata de un estudio basado en una investigación en curso sobre las transformaciones en la comunicación pública que están influyendo en el orden social en la era de la globalización.

El objetivo del presente artículo es presentar el planteamiento teórico y metodológico de dicha investigación. En concreto, se persiguen dos objetivos:

- En primer lugar, mostrar la pertinencia de un estudio en el momento actual, es decir, por qué se puede y debe analizar el papel de la comunicación en el orden social.

- Y, en segundo lugar, explicar la manera en que se está llevando a cabo ese análisis y la procedencia de los datos empleados.

Los resultados de la I+D: "La Producción Social de la Comunicación y la Reproducción Social en la Era de la Globalización" , que está realizando el grupo de investigación de la Universidad Complutense de Madrid "Identidades sociales y comunicación", proporciona unos datos que se consideran de gran pertinencia para el análisis del objeto de estudio que nos ocupa. Dicha I $+\mathrm{D}$ ha sido diseñada por el Catedrático de Teoría de la Comunicación Dr. Manuel Martín Serrano.

1 Es una investigación financiada por el Ministerio de Educación español, a través de convocatoria competitiva de I + D y que se está llevando a cabo por el Grupo de investigación "Identidades sociales y comunicación" de la UCM, del cual soy miembro. A partir de ahora será mencionada en el artículo como $<\mathrm{I}+\mathrm{D}>$. 
La estructura de este artículo se compone de cuatro partes: en la primera se justifica la pertinencia del objeto de la investigación así como un breve estado de la cuestión. En la segunda parte, se exponen los fundamentos metodológicos de la investigación, en relación con el planteamiento de la $\mathrm{I}+\mathrm{D}$ de la que procede. En la tercera parte se explican los aspectos teóricos que enmarcan la I+D y el presente estudio. En la última parte, se presentan las expectativas de este estudio a modo de conclusión.

\section{Estado de la cuestión y justificación del objeto de estudio}

Con este estudio se pretende contribuir al conocimiento de los cambios que han producido y continuarán haciéndolo las dinámicas [comunicación pública $\leftrightarrow$ consenso/conflicto social] en la sociedad actual, globalizada y sustentada por las TIC.

El funcionamiento de la comunicación pública está dando lugar a diversas transformaciones relacionadas con la globalización, tal y como se muestra a continuación:

- Ya se puede comprobar la notable influencia que los cambios en los sistemas comunicativos tienen sobre las dinámicas sociales: los nuevos sistemas informático-comunicativos ofrecen otras modalidades diferentes de acceso a los públicos y de interacción colectiva.

- Además, durante la precedente etapa histórica (época de las sociedades de masas y los medios de comunicación de masas) las formas establecidas de utilizar los medios de comunicación en la preservación del consenso social y la prevención de conflictos, eran más eficaces de lo que lo son actualmente.

Estas transformaciones en las opciones y el uso de las TIC, por un lado, y el contexto socio-económico y cultural, por otro, hacen pertinente que se contextualice y amplíe la investigación de este objeto de estudio.

Teniendo en cuenta que la comunicación pública puede generar diversos efectos y de orientación alternativa, los usos de la comunicación en las políticas públicas también debieran de ser distintos. Es por eso que la importancia del tema objeto de estudio se corresponde una de las funciones que debe cumplir la comunicación pública para la reproducción social (Cfr.: Martín Serrano, 2007): el papel de instancia mediadora en el mantenimiento del consenso social y, eventualmente, en la prevención o tratamiento de los conflictos sociales.

Martín Serrano (2004:88) señala: "la comunicación pública es una de las actividades colectivas destinadas a proveer de la información necesaria para la reproducción de la comunidad". Este es el objetivo concreto de este estudio: analizar el uso de la comunicación pública en cuanto afecta al consenso y al conflicto, para asegurar la reproducción del sistema social. Para ello, en este estudio se analiza la innovación comunicativa, sus efectos en la sociedad y los grupos afectados, para determinar qué resultados ofrecen etas innovaciones respecto al consenso y al conflicto social, teniendo en cuenta que la comunicación pública participa con ambas funciones 
mediadoras en la reproducción y el cambio social; concretamente, con las representaciones que ofrece de los nuestros y de los otros, de lo nuestro y de lo de los otros.

La sociología del conflicto y del consenso social ha sido muy prolífera desde el pasado siglo. La bibliografía al respecto es amplísima y no se puede citar en este articulo por cuestiones de espacio; sin embargo, un sociólogo español ha hecho el esfuerzo de aglutinar los principales aportes teóricos al respecto durante dicha época, en un capítulo titulado "El conflicto social" (González Seara, 1971:245-277), en el que el autor señala que se puede entender el orden social, de forma muy simplificada o resumida, desde dos visiones que han marcado las teorías sociológicas clásicas y contemporáneas sobre el conflicto y el consenso social:

- La visión de Russeaau, que plantea la cohesión social como el consenso libre y universal, el acuerdo general de todos.

- Sin embargo, Hobbes considera la cohesión social como sinónimo de coacción por parte de los que ostentan el poder, quiénes disuaden a los dominados de tentativas de adquirir dicho poder.

Estas dos perspectivas han marcado dos líneas bien diferenciadas que abordan el objeto de este estudio: la del consenso, defendida por Comte y Durkheim, quienes consideran esencial la armonía social basada en el consenso general entre individuos; y la línea del conflicto como motor del cambio histórico, sostenida por el marxismo y el darwinismo social.

Siguiendo a González Seara (1971), es necesario remarcar la influencia que las teorías marxistas del conflicto ejercen sobre la línea darwinista, en la que destacan sociólogos europeos como Spencer, Gumplowicz o Ratzenhofer, entre otros, y los americanos como Sumner, Simmel o von Wiese. Cooley y Ross "ven en el conflicto algo esencial y positivo para las relaciones sociales" (González Seara, op.cit., p. 250), del mismo modo que lo contemplan Ward, Samall o Robert Park. También son importantes las aportaciones de Weber y de Pareto, para los que el conflicto es algo fundamental en las relaciones sociales.

Respecto a los sociólogos occidentales contemporáneos, destacan autores como Talcott Parsons, Eltosn Mayo, Lloyd Warner, Kurt Lewin, George Lundberg o Robert Merton, entre otros, a quienes González Seara (op.cit., p. 252-253) designa como los representantes del análisis estructural-funcional, preocupados por el orden social y que consideran el conflicto como una conducta desviada.

La sociología moderna del conflicto se sitúa frente a esta línea de análisis, con Dahrendorf como uno de los más importantes representantes, autor de una teoría del conflicto social que excluye la utopía de una sociedad en perfecto funcionamiento. También destacan Van Den Berghe, Galtung, Aron, Mills y Coser; éste último, sigue esencialmente las ideas de Simmel, y su obra imprescindible para este objeto de estudio es The functions of social conflict, 1956 (citado en González Seara, 1971). 
En la actualidad, parece haber unanimidad en cuanto a que los conflictos surgen constantemente; incluso, con la Era de la Globalización, éstos adquieren nuevas formas y por eso conviene analizar cómo se enfrenta a ellos la sociedad, es decir, hay que considerar el conflicto como una parte positiva de la sociedad misma que tiene que resolver de manera constante. "El conflicto no desaparece, sino que varían los mecanismos para su resolución" (González Seara, op.cit., p 269).

Dado que parece muy productiva y esclarecedora la tradición de estudios estudios sociológicos sobre el consenso y el conflicto, se considera imprescindible la lectura y análisis de las obras que los autores mencionados han dedicado al orden social. Pero, tal y como se ha señalado, es una tarea que se adecua a otro espacio y tiempo.

Además, la aproximación al objeto material en el estudio que se presenta en este artículo es, particularmente, a través de la comunicación. Las formas en las que la comunicación pública opera (o deja de operar) sobre el consenso y los conflictos sociales, ha sido un tema de estudio recurrente por la psicología social desde los trabajos de Le Bon y de Tarde, cuando analizaron los conceptos de "masa" y "público"; respectivamente.

En 1895, Gustave Le Bon ya señalaba cómo la prensa escrita había dejado de producir objetos de consenso ideológico entre las masas, para reproducir las opiniones de las multitudes.

La prensa, directora de la opinión de nuestra época, ha tenido que ceder, lo mismo que los gobiernos, ante el poder de las muchedumbres (...). Se ha convertido en simple agencia de información, renunciando a imponer ideas ni doctrina (Le Bon, 1968: 160).

Gabriel Tarde plantea, por su parte, una secuencia de oposiciones a nivel social en la que, en un primer nivel, se situaría el choque (conflicto) o guerra, que es la forma primaria y más frecuente entre los pueblos "poco cultos"; en un segundo nivel, estaría la competencia o rivalidad económica que presentan las sociedades democráticas (sociedades industriales), puesto que es la forma dominante que presenta la oposición; y en un tercer nivel, la discusión o el debate, en las formas características que se manifiestan en las sociedades democráticas.

Naturalmente, esta sucesión de formas de la oposición depende de los cambios e intereses de los individuos, de la distancia social entre las clases, así como de la facilidad de las comunicaciones (Tarde, 1986:26).

El interés por entender el papel de la comunicación en la gestión del consenso y el control de los conflictos ha llegado hasta nuestros días, con autores que identifican una clara relación entre la producción de noticias (selección y narración del acontecer) y el establecimiento de estados de opinión y consenso que interesan a las clases dirigentes. De entre estos autores, europeos y norteamericanos, es preciso detenerse en las aportaciones de Noam Chomsky, Edward S. Herman, Ignacio Ramonet, Herbert Schiller, Armand Mattelart y otros autores englobados, generalmente, en los estudios críticos sobre la comunicación. 
Por ejemplo, Chomsky considera que la toma de decisiones en nuestras democracias capitalistas no las toma el pueblo sino las "manos privadas" o "instituciones ideológicas que canalizan el pensamiento y las actitudes dentro de unos límites aceptables, desviando cualquier reto en potencia hacia el privilegio y la autoridad establecidos" (Chomsky, 1992:8). Además de su conocido modelo de propaganda, escrito junto con Herman, Chomsky ha dedicado parte de su obra a detectar mecanismos de manipulación y creación de consenso, sobre todo por parte de los gobiernos norteamericanos en los últimos cincuenta años (Chomsky y Herman, 1994).

Desde los años setenta, Armand y Michèle Mattelart vienen presentando un planteando parecido cuando señalan que los ciudadanos asumen determinados valores y visiones del mundo como consecuencia de la internacionalización y creciente importancia de los medios y sistemas de comunicación (Mattelart, 1987).

Por lo tanto, el hilo común que une las aportaciones de todos estos autores es su denuncia ante el uso de la comunicación por parte de los gobiernos y otros poderes establecidos para mantener el status quo. Empero, es preciso subrayar que estas denuncias están generalmente referidas a acontecimientos o países concretos, desde los años sesenta hasta hoy.

Sin embargo, son muchos menos los estudios que se han preguntado por las relaciones entre la comunicación y el orden social a un nivel sociohistórico. Es decir, qué cosas van cambiando (o tienen que ir cambiando) en la comunicación pública, en periodos de tiempo históricos, para poder seguir manteniendo el orden social establecido.

$Y$ es, precisamente, este el nivel en el que está ubicada esta investigación, puesto que parece ser el adecuado para comprender qué cosas de la comunicación han cambiado o tenido que cambiar en la actual etapa histórica de la globalización, para que, en un mundo cada vez más conflictivo, se mantengan diversos consensos sociales básicos.

Por consiguiente, es posible seguir ampliando el campo de estudio en este nivel de análisis macrosociológico. El enfoque sociohistórico y macro elegido para esta investigación ya ha sido trabajado por teóricos de la comunicación que se han encargado de los vínculos históricos entre lo que cambia en la comunicación y lo que cambia en la sociedad. Por ello, tal y como se mostrará en el epígrafe cuarto, se considera de especial relevancia la tradición de estudios desarrollada, entre otros, por Martín Serrano desde los años setenta, primero en La Mediación Social (1977) y luego en La Producción Social de Comunicación (1986).

\section{Metodología}

En el presente estudio se están utilizando las categorías y modelos de análisis de la Teoría de la mediación social, que son los mismos que el grupo de investigación 
"Identidades sociales y comunicación" viene implementando en un gran repertorio de investigaciones. El objetivo principal de esta I+D es el conocimiento de las representaciones de los fenómenos, que relacionan las transformaciones de la comunicación con los cambios sociales, con el fin de conocer cómo funcionan los mecanismos de reproducción social en la era de la globalización, que ejercen las instituciones mediadoras de comunicación pública, más concretamente, a través de sus relatos especializados en información científico-técnica.

Para poder conocer esas representaciones, Martín Serrano diseña una tipología de análisis de contenido basada en el análisis de estructuras lógicas. Se trata de una metodología original del Autor, frecuentemente probada y aplicada desde mediados de los ochenta en análisis de contenido por el autor y sus discípulos. La aplicación de análisis lógicos permite, entre otras utilizaciones, identificar los códigos que se encuentran en los productos culturales, mediante los cuales se comparten, se comunican y extienden, las representaciones, visiones o modelos del mundo. Son las instituciones mediadoras las que reproducen dichos modelos de orden para el control social, principalmente a través de códigos que organizan los relatos y, sólo de forma secundaria, mediante los contenidos que aparecen expresos en esos relatos (Martín Serrano, 1977:90).

Esta metodología requiere de un trabajo previo de recogida de información de un número significativo de textos de carácter técnico-científico que traten sobre los fenómenos cuyas representaciones se quieren conocer.

Dada la cantidad ingente de información manejada en la I+D y, para facilitar una posterior explotación, se ha procedido a recoger las referencias mencionadas sistemáticamente, y se ha registrado: por un lado, la fuente institucional o fuentes institucionales a las que se les imputaba la información; y, por otro lado, los datos, colocándolos en lugares predeterminados, para así construir sentencias lógicas que se constituirían en las unidades de análisis del presente estudio. Para ello, se ha creado una aplicación informática creada por el miembro del Grupo de investigación, profesor Dr. Javier Martin, que reproduce un protocolo de análisis diseñado por el Profesor Dr. Manuel Martin Serrano para la I+D.

Es preciso destacar que, en dicho protocolo, hay unos apartados específicamente referidos a transformaciones de la Comunicación Pública que tienen incidencia en el consenso y en los conflictos sociales, y que ya están recogidos y volcados en la aplicación. De esta forma, el material que se maneja en la presente investigación está compuesto por un total de tres mil sentencias que se han constituido siguiendo el modelo de explotación de resultados mencionado.

Por lo tanto, este estudio se nutre de una importante base de datos que la I+D ofrece a los investigadores y académicos, con una gran variedad de información, además de importantes recursos metodológicos ya implementados (metodologías de análisis de contenido lógicas, discriminativas y estructurales $)^{1}$. La presente investigación, selecciona de dicha base la información que tiene pertinencia y opera con los mismos modelos y programas. El trabajo que se está realizando en este estudio 
actualmente, consiste en la clasificación de la información relevante para el objeto de estudio para su posterior análisis.

Este análisis, parte del siguiente supuesto, formulado por el Profesor Dr. Manuel Martín Serrano, en el diseño de dicha I + D: "Si se excluyen las medidas de fuerza, el orden social resulta del establecimiento de los consensos que les permitan a los miembros de un colectivo, manejar los disensos (conflictos, discrepancias...) sin que se pierda la cohesión ni el sentimiento de pertenencia a la comunidad".

\section{Mecanismos de reproducción social en la era de la globalización: principios teóricos}

El planteamiento de este estudio, que abarca una de las dimensiones del proyecto I+D se beneficia de un diseño que es, a) macrosociológico, b) sociohistórico y c) orientado a la transferencia de los resultados:

- La propuesta se ajusta a ese enfoque macrosociológico puesto que se relaciona: [comunicación pública $\leftrightarrow$ (consenso y conflicto social)] $\rightarrow$ globalización

- Se está realizando un estudio de "las transformaciones de la Comunicación pública en relación con "los cambios correlativos en las dinámicas sociales del consenso y del conflicto"2 (Martín Serrano, 1978a). De esta forma, también se aplica el enfoque sociohistórico que proporciona la I+D.

Esto es lo que hace propicio en este estudio llevar a cabo análisis prospectivos como los que se están implementando en la investigación de referencia, con el objeto de conseguir realizar previsiones referidas a los cambios esperables en las dinámicas del consenso y de los conflictos sociales.

- El diseño está orientado a la transferencia de los resultados a las políticas públicas.

Es decir, tal y como se indica en el apartado anterior, el proyecto I $+\mathrm{D}$ y, por ende, el objeto de estudio de este artículo, se inscriben dentro de un marco teórico fundamental de estudios encabezados por las teorías recogidas en La Mediación Social (1977) y La producción social de comunicación (1986), de Manuel Martín Serrano. En La mediación social, el autor presenta en el capítulo "Modelos generales que explican el ajuste al cambio social", los modelos de tipo conflictivo, típicos de una sociedad monopolista ${ }^{3}$. Respecto al orden social, Martín Serrano señala lo siguiente:

En la medida en que las contradicciones entre las innovaciones y las normas no desembocan en un conflicto que cuestione la totalidad del orden social, se prueba que los controles sociales actúan cortando los lazos de interdependencia existentes entre innovación, normas y organización social (Martín Serrano, 2008:61).

De este modo, el planteamiento teórico de La Mediación Social se basa en las contradicciones entre innovación tecnológica y cambio cultural. 
Cuando la sociedad se ve sometida a la presión de constricciones surgidas de la innovación, incompatibles con las constricciones que presionan desde la cultura, se encuentra en una situación disonante (Martín Serrano, 1977:44).

Teniendo en cuenta la perspectiva dialéctica, una sociedad en esta situación puede superar la contradicción o negarla. En el primer caso solo cabe la superación a través del cambio mediante un proceso revolucionario mientras que, en el segundo caso, tiene que establecer el mejor ajuste posible entre innovación tecnológica y cambio cultural para que el sistema se pueda seguir reproduciendo en estado de contradicción. De esta forma, Martín Serrano afirma lo siguiente:

La sociedad que así funciona se mantiene porque posee sistemas de ajuste que hacen posible que los actores sociales se adapten a vivir en un estado de crisis permanente, sin cuestionar el sistema global (Martín Serrano, 1977:20).

En esta obra el autor indica que lo que está ocurriendo en las sociedades capitalistas postindustriales es que, para hacer frente a los desajustes, "se espera que cada individuo «se cambie» cuantas veces sea necesario y tanto como resulte preciso" (Martín Serrano, 1977:21).

A tal objeto se destinan muchos recursos sociales: a actividades mediadoras (que "requieren inversiones en infraestructuras, servicios y bienes para la mediación social") con un uso reproductivo, necesarias (y a la vez productivas) para que perdure el modelo socioeconómico. El autor añade: "La gestión del ajuste colectivo reproduce consensos al tiempo que produce beneficios" (Martín Serrano, 1977:21). En este estudio se investiga, precisamente, los usos de la Comunicación Pública como instancia mediadora que se utiliza para la reproducción de consensos que permitan dicho ajuste colectivo.

En La producción social de la comunicación (1986), el autor describe las operaciones de mediación que realizan los medios de comunicación pública (Martín Serrano, 2004:162-164), con el fin de preservar el consenso y manejar los conflictos sociales:

- la mediación cognitiva, "entre la aparición de nuevos aconteceres, cuya producción compromete el consenso social, y la reproducción de normas y valores socialmente compartidos (conflicto entre aconteceres y creencias)", y

- la mediación estructural, "entre la imprevisibilidad de los nuevos emergentes ${ }^{4}$ y la previsión de la programación del medio (conflicto entre aconteceres y formas de comunicación de los medios)".

Son estos consensos y conflictos sociales a los que se refiere el autor, los que se espera categorizar en este estudio a partir del material que se está extrayendo de la bases de datos de la $\mathrm{I}+\mathrm{D}$, para su posterior análisis. 


\section{A modo de conclusión}

El primer análisis ejecutado en el estudio que se presenta en este artículo incluye la revisión de mil quinientas sentencias lógicas. Puesto que la investigación se encuentra en una fase inicial, todavía no se dispone de las tipologías de consenso y conflicto social que podrían extraerse de esta base de datos en relación con los diversos criterios de análisis. Sin embargo, ya se vislumbra un escenario que configura la manera en la que se está analizando teóricamente el conflicto y el consenso en relación con las transformaciones de las TIC.

Se va a tomar en consideración las siguientes opciones para el análisis: por un lado, conflictos relacionados con la comunicación que se han tratado hasta ahora y forman parte la tradición sociológica, y nuevos conflictos generados por las transformaciones en el sistema de comunicación; por otro lado, las distintas áreas en las que las transformaciones del sistema de comunicación genera consenso.

Una vez se haya finalizado con el análisis de todas las sentencias lógicas que ha proporcionado la I+D "La Producción Social de la Comunicación y la Reproducción Social en la Era de la Globalización”, la siguiente fase consistirá en la explotación de los datos obtenidos.

En la medida en que este estudio contribuya a objetivar las relaciones entre cambio, consenso y conflicto sociales y las distintas maneras en que los especialistas hablan de ello, se espera que esta propuesta contribuya a sistematizar el planteamiento de la cuestión y a desarrollar propuestas y alternativas, principalmente teóricas, eventualmente metodológicas, y en cualquier caso sociopolíticas, con el fin de disminuir las dimensiones disfuncionales que puedan tener los cambios comunicativos en el momento histórico en que se están produciendo.

\section{Bibliografía}

\subsection{Libros}

CHOMSKY, N. (1992): Ilusiones necesarias: control del pensamiento en las sociedades democráticas. Madrid: Libertarias/Prodhufi.

CHOMSKY, N. y HERMAN, E. S. (1994): Manufacturating Consent. The Political Economy of the Mass Media. Londres: Vintage.

GONZÁLEZ SEARA, L. (1971): La sociología, aventura dialéctica. Madrid: Tecnos. LE BON, G. (1968): Psicología de las multitudes. Buenos Aires: Albatros.

MARTÍN SERRANO, M. (2008) (1 ${ }^{\text {a }}$ Ed. 1977): La mediación social. Madrid: Akal. MARTÍN SERRANO, M. (2004) ( $1^{\mathrm{a}}$ Ed. 1986): La producción social de comunicación. Madrid. Alianza. 
MATTELART, A. y MATTELART, M. (traducción, notas y aportaciones bibliográficas de Gilles Multigner) (2000) (Edición original, 1987): Pensar sobre los medios: comunicación y crítica social. Santiago de Chile: LOM 2000

TARDE, Gabriel (1986): La opinión y la multitud. Madrid: Taurus Ediciones.

6.2 Artículos en publicaciones web

MARTÍN SERRANO, M. (1978a). Introducción del libro "Métodos actuales de investigación social". En: Métodos actuales de investigación social. Madrid: Akal. p. 7-11.

MARTÍN SERRANO, M. (1978b). "Un método lógico para analizar los significados. Aplicación al estudio del lenguaje de la TVE. En: Reis (Revista Española de Investigaciones Sociológicas), ${ }^{\circ}$ 2. p. 21-51. Disponible en: http://eprints.ucm. es/13256/. Consultado el 20 de marzo de 2013.

MARTÍN SERRANO, M. (1985). "Mediación cognitiva y estructural". Extraído de "La mediación de los medios de comunicación". En: Morgas, Miquel de (ed.): Sociología de la comunicación de masas. I. Escuelas y autores. Barcelona: Gustavo Gili, p. 141-162. Disponible en: www.epints.ucm.es . Consultado el 14 de marzo de 2013.

MARTÍN SERRANO, M.(1989): "Diseños para investigar la producción social de comunicación". En: Reis (Revista Española de Investigaciones Sociológicas), $\mathrm{n}^{\circ}$ 48. p. 79-90. ISSN 0210-5233. Disponible en: http://eprints.ucm.es/13147/. Consultado el 21 de marzo de 2013.

MARTÍN SERRANO, M. (2007). "Prólogo para La Mediación Social en la era de la globalización" En: Mediaciones Sociales. Revista de Ciencias Sociales y de la Comunicación, $\mathrm{n}^{\mathrm{o}}$ 1, segundo semestre de 2007, p. 1-24. ISSN electrónico: 19890494. Universidad Complutense de Madrid. Disponible en: www.epints.ucm.es . Consultado el 3 de julio de 2013.

\section{Notas}

1 Las características de estas metodologías están descritas en los documentos que ha depositado en el repositorio abierto de e-prints, el Manuel Martín Serrano, autor del diseño de la investigación: "Un método lógico para analizar los significados" (1978b) (http://eprints.ucm.es/13256/); y "Diseños para investigar la producción social de comunicación" (1989) (http://eprints.ucm.es/13147/).

2 Dentro del ámbito que acota la investigación de [comunicación pública $\leftrightarrow$ ( consenso y conflicto social) ] $\rightarrow$ globalización, se toma en cuenta: lo que está transformándose, va a transformarse, puede transformarse; lo que está cambiando, va a cambiar, puede cambiar; lo que no existía y ahora existe, va a existir o puede existir; lo que no pasaba y ahora pasa, va a pasar, o puede pasar; lo que no se podía y ahora se puede, se va a poder. Véase MARTÍN SERRANO, M. (1978a). Introducción del libro "Métodos actuales de investigación social". En: Métodos actuales de investigación social. Madrid: Akal. p. 7-11.

3 El término monopolista se corresponde con la clasificación de Marx en las etapas. En nuestro contexto actual sociedad globalizada corresponde con lo mismo. Tal y como explica Martín Serrano 
en La mediación social, se entiende por sociedad monopolista aquella que los sociólogos no marxistas definen con rasgos propios de la "sociedad posindustrial», "sociedad de consumo de masas», «sociedad del bienestar», etc. En MARTÍN SERRANO, M. (2008). La mediación social. Madrid: Akal. p. 41.

4 Véase la definición que ofrece Martín Serrano cuando se refiere al concepto de "acontecer público" de "emergente": "Algo que ocurre (o deja de ocurrir) en el entorno, en cualquier lugar y en cualquier tiempo, cualesquiera que sean sus causas y efectos". En MARTÍN SERRANO, M. (2004), La producción social de comunicación. Madrid: Alianza editorial. p. 146.

\section{La autora}

Belén Casas-Mas es Doctoranda en Comunicación Social por la Universidad Complutense de Madrid, y becaria FPU (Formación de Profesorado Universitario) por el Ministerio de Educación, Cultura y Deporte, en el Departamento de Sociología IV de la Facultad de Ciencias de la Información (UCM). Actualmente, forma parte del Grupo de investigación "Identidades sociales y comunicación" de la UCM. 